\title{
A Simultaneous Wideband Impedance Matching and Bandpass Filtering Technique using NUTL Segments at $15 \mathrm{GHz}$
}

\author{
Rana A. Shaheen, A. Sethi, R. Akbar, *Janne P. Aikio, T. Tuovinen, *T. Rahkonen, A. Pärssinen \\ Centre for Wireless Communication - Radio Technologies, * Electronics Laboratory - Circuits and \\ Systems, University of Oulu, Finland
}

\begin{abstract}
This paper presents a simultaneous high-Q impedance matching and bandpass filtering technique for low-noise amplifiers (LNA) for radio frequency integrated circuit (RFIC) phased array system at $15 \mathrm{GHz}$, over the bandwidth of $1 \mathrm{GHz} A$ non-uniform transmission line (NUTL) segment is used as a major building block, which provides feasible integration for multiple antennas. A feeding network for single element receiver is demonstrated. The simulated and measured results show sufficient impedance matching of input feeds with $\mathrm{S} 11<-10 \mathrm{~dB}$ over a relative bandwidth of $7 \%$ at $15 \mathrm{GHz}$
\end{abstract}

Index Terms - bandpass filtering, LNA, input matching, phased array.

\section{INTRODUCTION}

In order to reach extremely high data rate targets up to 10 Gbits/s for fifth generation (5G) communication systems the contiguous and broader bandwidth (BW) would be needed [1]. Due to the crowded spectrum below $6 \mathrm{GHz}$, the increasing interests are on sub-millimeter (sub-mmWave) and mmWave regions [2]. A higher relative BW at sub-mm wave frequencies alone set practical constraints for radio frequency (RF) implementation in addition to the demanding system specifications. This work focuses on the receiver side where the noise performance highly relies on low-noise amplifier (LNA), which needs to contribute the minimum noise with the necessary voltage gain. For systems at cm-wave or mm-wave frequencies, with relative BW close to $10 \%$ of center frequency, one of the design challenges is to match the LNA input sufficiently to $50 \Omega$ for the entire BW. Particularly in RFIC design, due to the impacts of parasitic components from the structures such as electrostatic discharge (ESD) diodes, the LNA input impedance typically appears to be highly reactive resulting in high-Q impedance. That makes impedance match over the very wide BW complicated. The problem gets even more severe for the typical LNA topology with common source input. In this paper, we propose a method of matching the high-Q impedance for a wide $\mathrm{BW}$ of $1.2 \mathrm{GHz}$ at a high centre frequency of $15 \mathrm{GHz}$ (relative $\mathrm{BW}$ of 7\%) using NUTL segments. The topology is utilized to match the RF-input of a beam steering test chip, designed for 15 $\mathrm{GHz}$ center frequency.

\section{MOTIVATION}

A common source inductively degenerated (CSID) LNA is typically used for narrowband systems [3]. The capability to achieve low NF and input matching simultaneously, is the main motivation for using this topology. The input impedance of a CSID amplifier is given by [3]

$$
Z_{\text {in }}=s\left(L_{\mathrm{g}}+L_{\mathrm{s}}\right)+\frac{1}{s C_{\mathrm{gs}}}+\frac{g_{\mathrm{m}}}{C_{\mathrm{gs}}} L_{\mathrm{s}},
$$

where $\mathrm{Lg}$ is the inductance added at the input of LNA, Ls is inductance at the source terminal of the input transistor, $\mathrm{Cgs}$ is gate to source parasitic capacitance and gm is transconductance of input transistor. The CSID LNA has typically input impedance presented by (1), although the parallel capacitance of the ESD turn Zin capacitive. In (1), $\mathrm{Lg}$ is used to cancel capacitive part, and Ls is gyrated to cause a resistive Zin, usually tuned directly to $50 \Omega$ impedance. Different techniques are used for realizing $\mathrm{Lg}$ or resonant circuits, such as lumped components or transmission lines (TL). Due to the fact that the pad size and the pitch between the pads in RFIC is in sub mm-range, the use of TLs for input matching is inevitable for multiple antenna systems. NUTL are used for impedance matching, resonator and filter design techniques [4]. Low pass and bandpass filter design techniques using different shapes of NUTLs are presented in [4]. A method of optimization of minimum reflection co-efficient of an impedance transformer using varying TLs is demonstrated in [5] along with multiband filter design. Due to the wider passband response and their integration flexibility in denser networks, taper line segments could become potential candidates for input feedlines of phased array systems providing input match as well as filtering, simultaneously.

Cancellation of reactive part is usually designed already in chip. However, if this cannot be implemented, the high$\mathrm{Q}$ input impedance upon matching provides additional benefit of very sharp bandpass filtering $(<-20 \mathrm{~dB} /$ decade) to input signal. In this work, we utilize the opportunity to use external wideband matching technique using NUTLs for matching relatively high-Q input impedance of phased 


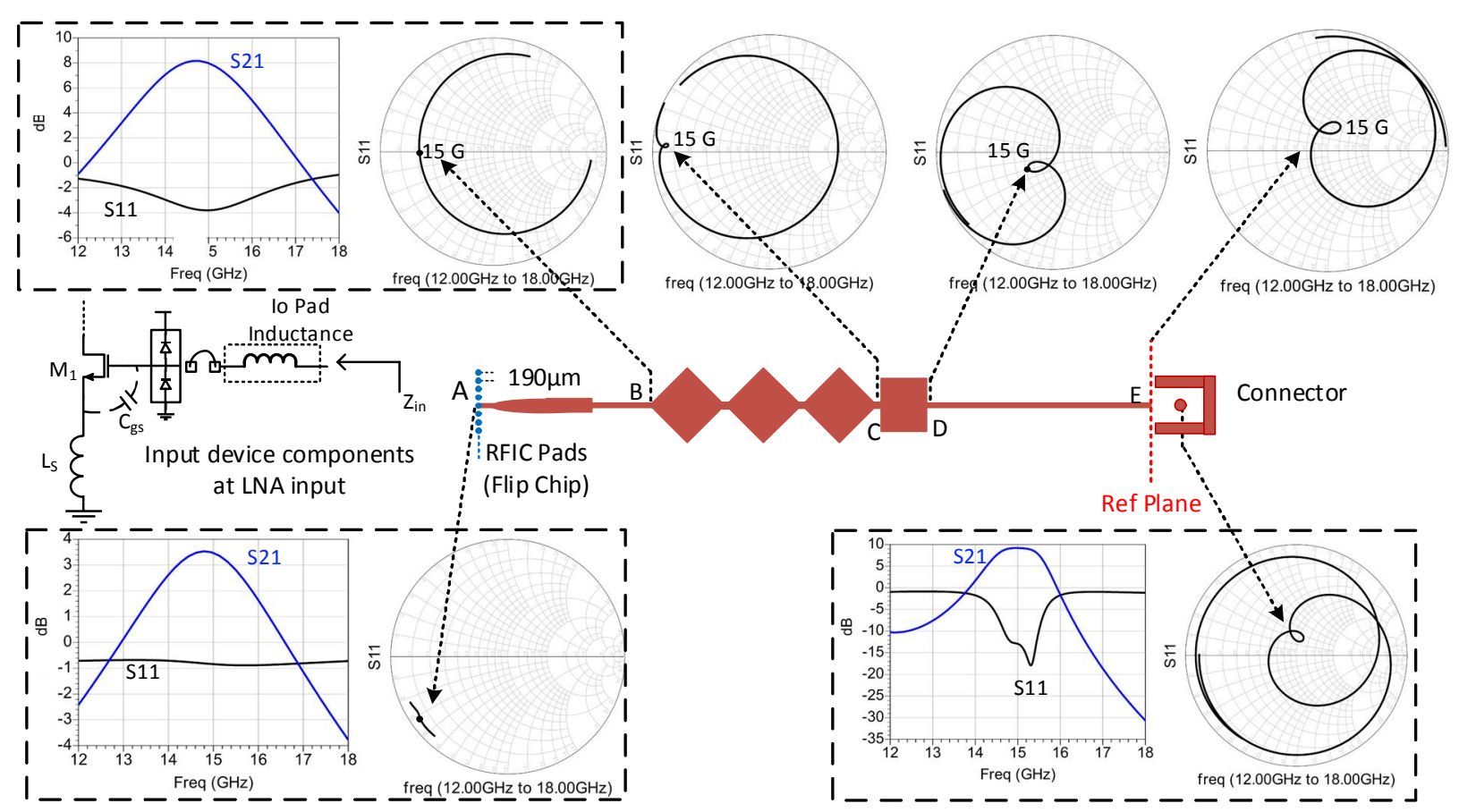

Fig 1. Schematic of input device with the design procedure of impedance matching line showing S11 and S21 (Simulations) graphs at key interfaces.

array inputs, which also relaxes the additional filtering requirements. According to the best knowledge of authors, this is the first time that a matching technique is reported for achieving simultaneously high-Q wider BW input match and bandpass filtering. The detailed design procedure is discussed in section III.

\section{DESIGN PROCEDURE}

The designed RFIC has eight RX inputs placed next to each other on the same edge of the IC. The chip is attached to PCB as a flip chip via solder bumps. As this is a nonpackaged flip-chip, the chip pad areas dictate the pitch. The pitch between two consecutive IO pads is $190 \mu \mathrm{m}$ with the pad area of $100 \mathrm{um} \times 100 \mathrm{um}$. With such small pad dimensions, a $0.1 \mathrm{~mm}$ wide transmission line is needed to feed the signal to the input of the LNA. A four-layer PCB was used with the material characteristics of $\varepsilon r=2.99$ and losstan $\delta=0.0017$. Total thickness of PCB is $0.43 \mathrm{~mm}$ resulting in the linewidth of $230 \mathrm{um}(50 \Omega)$ with the top metal. Impedance matching of a single receiver at center frequency of $15 \mathrm{GHz}$ is achieved in three steps, discussed as follows.

\section{A. Transformation of Capacitive Reactance}

At the targeted centre frequency, the input impedance at input pad of LNA appears to be highly capacitive, as shown in Fig. 1 by point ' $A$ '. In order to cancel the reactance, the initial TL with the width of $0.1 \mathrm{~mm}$ together with a taperedline is provided. The long taper line at the beginning is mandatory as it spans out the inputs, providing sufficient space between them. The main goal of the initial transformer is to transform the reactive impedance close to low ohmic real plane on the Smith chart, shown in Fig.1 by graphs at point ' $\mathrm{B}$ '.

\section{B. Taper-Line Diamond Segments for Multiple Resonances}

This part is the core of the matching circuit. A $\lambda / 4$ long single segment rotates the impedance of targeted bandwidth into a circle as shown by graphs at interface ' $\mathrm{C}$ ' in Fig. 1. By adding multiple sections, the impedance circle is narrowed so that it can be later on shifted to the centre of the Smith chart. Three segments are used, which results in two rotations of impedance points in the Smith chart. One diamond-shaped segment is realized by combining two taper lines. The characteristic impedance of the segment varies linearly from $50 \Omega$ to $8 \Omega$ and again back to $50 \Omega$.

\section{Impedance Transformer for Shifting Resonance}

The purpose of this section is to transform the impedance response to the centre of the Smith chart. This is carried out with the impedance step transformers starting with a $\lambda / 8$ line segment ( $Z 0$ of $10 \Omega$ ), which provides rotation in the impedance response at frequencies of interest (plot at 
interface ' $D$ ' in Fig. 1). A $50 \Omega$ TL is used until interface ' $E$ ' (reference plane for connector model) in Fig. 1, which rotates frequency impedance points in counter-clockwise direction, i.e. towards center. Finally, connector model is added (plots at connector interface in Fig. 1). This rotation of S11 around center, provides bandpass-type response in S11 and S21 plots as shown in Fig. 1 at connector interface.

\section{RESULTS}

Fig. 1 at connector interface, shows the final S11 responses on Smith chart and the magnitude plot. It is visible that at center frequency, S11 has bandpass filtertype response with the $\mathrm{S} 11<-10 \mathrm{~dB}$ for more than $1 \mathrm{GHz}$ bandwidth. The results are obtained from electromagnetic (EM) simulations with ADS Momentum tool, using RC parasitic extracted simulated s11-parameters of the IC as a load. A single element LNA is implemented on the RFIC and the PCB design was carried out on the four-layer PCB for the characterization of the system as shown in Fig. 2.

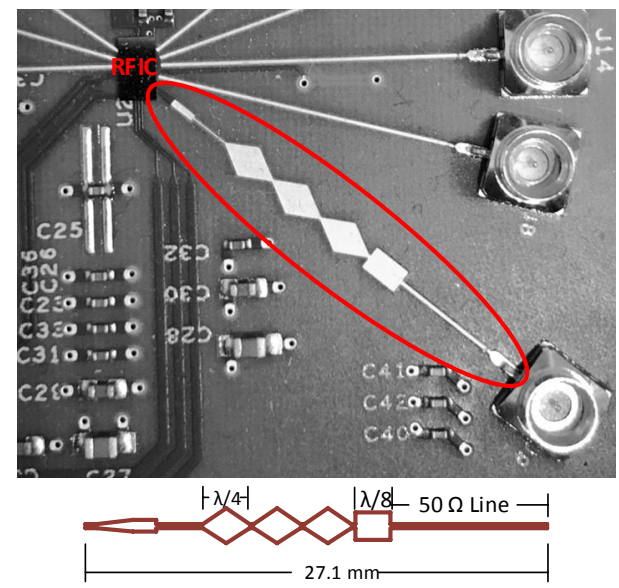

Fig 2. Designed trace implemented on PCB test board including RFIC

The LNA input feed lines are designed by using the above-mentioned procedure such that each line has the same length and impedance transformer design. The microstrip bends are used to realize angle for different lines. The design of taper-line diamond segments provide great flexibility for integrating multiple antenna system on a PCB. Measurement result of a single channel input return loss is shown in Fig.3. Measured results follows the filter function according to the simulation. While estimating input impedance of LNA, only RC parasitic extraction was used. Since, RC extraction method does not take into account the inductance of conductors at LNA input pads, which may have impact on input impedance at $15 \mathrm{GHz}$. However, the resonance is moved to $15.6 \mathrm{GHz}$.

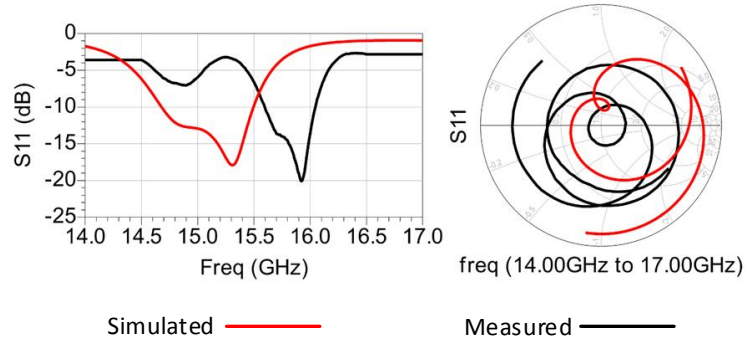

Fig 3. Simulated and measured input return loss including connector model

\section{CONCLUSION}

A method is presented in this paper to demonstrate simultaneous wideband impedance match and bandpass filtering for LNA inputs in RFIC phased array systems. A NUTL segment is used as unit building block and two impedance transformers. In addition to achieve good input matching, i.e. S11<-10 dB, bandpass filtering $(-20$ $\mathrm{dB} /$ decade) across $1.2 \mathrm{GHz} \mathrm{BW}$ provides additional benefit for the structure. High-Q impedance upon matching for wider BW, always comes for the cost of some losses. Total loss of the structure was estimated as $1.5 \mathrm{~dB}$. The designed structure provides better solution for implementing input feed lines for LNAs in phased array systems with additional characteristics of bandpass filters.

\section{ACKNOWLEDGEMENT}

The authors would like to thank Nuutti Tervo, Saila Tammelin, Risto Vuohtoniemi, and Ville Hevosmaa for their technical support. Nokia Corporation is acknowledged for financial support and Global Foundries for silicon fabrication.

\section{REFERENCES}

[1] ITU-R Recommendation M.2083 "IMT Vision Framework and overall objectives of the future development of IMT

[2] W. Roh, J.-Y. Seol, J. Park, B. Lee, J. Lee, Y. Kim, J. Cho, K. Cheun, and F. Aryanfar, "Millimeter-wave beamforming as an enabling technology for $5 \mathrm{G}$ cellular communications: Theoretical feasibility and prototype results," IEEE Commun. Mag., vol. 52, no. 2, pp. 106-113, Feb. 2014.

[3] D. Shaeffer and T. H. Lee, "A 1.5-V , 1.5-GHz CMOS low noise amplifier," IEEE J. Solid-State Circuits, vol. 32, no. 5 , pp. 745-759, May 1997.

[4] M. L. Roy, and A. P'erennec, "The continuously varying transmission-line technique - application to filter design," IEEE Trans. Microwave, vol. 47, no. 9, Sep. 1999.

[5] H. Yao-wen, and F. K. Edward, "Direct synthesis of passband impedance matching with nonuniform transmission lines," IEEE Trans. Microw. Theory Tech., vol. 58, no. 4, Apr. 2010 